\title{
Atividade eletromiográfica dos músculos do joelho em indivíduos com reconstrução do ligamento cruzado anterior sob diferentes estímulos sensório-motores: relato de casos
}

\author{
Electromyographic activity of knee muscles in individuals with anterior cruciate \\ ligament reconstruction under different perturbations: case report
}

\author{
Jefferson Rosa Cardosol, Artur Inacio Prado2, Henrique Kiyoshi Iriya2, \\ Ana Beatriz de Almeida Noronha Santos, Hugo Maxwell Pereira ${ }^{4}$
}

\section{Fisioterapeuta; Prof. Dr. do Laboratório de Eletromiografia e Análise Cinemática do Depto. de Fisioterapia da UEL (Universidade Estadual de Londrina, Londrina, PR) \\ 2 Fisioterapeutas; Especialistas em Recursos Terapêuticos e Técnicas Posturais na UEL \\ 3 Graduanda em Fisioterapia (Bolsista de Iniciação Científica - CNPQ) na UEL \\ 4 Fisioterapeuta; mestrando em Educação Física no Programa Associado Universidade Estadual de Maringá-UEL}

ENDEREÇO PARA

CORRESPONDÊNCIA

Jefferson R. Cardoso

Av. Robert Kock 60

86038-440 Londrina PR

e-mail: jeffcar@hotmail.com

Artigo apresentado à UEL para obtenção do título de

Especialista em Recursos

Terapêuticos e Técnicas

Posturais dos autores 2
APRESENTAÇÃO ago. 2006

ACEITO PARA PUBLICAÇÃO dez. 2007
Resumo: O objetivo deste foi descrever a atividade eletromiográfica dos músculos estabilizadores do joelho de indivíduos que receberam diferentes enxertos na cirurgia de reconstrução do ligamento cruzado anterior (LCA). Foram avaliados dois casos de enxerto patelar, dois de enxerto posterior e dois controle, durante seis estímulos proprioceptivos ao indivíduo em apoio unipodal (em solo, prancha inclinada, prancha redonda, balancinho e rollerboard em sentido ântero-posterior, AP, e médiolateral, ML). O sinal eletromiográfico normalizado foi captado durante contração isométrica voluntária máxima (CIVM) de cada músculo (vasto medial oblíquo, vasto lateral, semitendinoso, bíceps femoral e gastrocnêmio). Ao comparar os músculos vasto medial oblíquo e vasto lateral entre os casos, foi encontrada porcentagem da CIVM maior na amostra com enxerto patelar durante os estímulos solo, prancha inclinada e rollerboard AP, enquanto nos casos de enxerto posterior isso ocorreu sob os estímulos de balancinho e prancha redonda. Ao analisar somente os músculos flexores, os sujeitos com enxerto posterior apresentaram maior atividade sob os estímulos de balancinho e rollerboard ML. Conclui-se que os casos com enxerto patelar ativam mais sua musculatura que aqueles com enxerto posterior; e a quantidade de ativação muscular parece variar para cada estímulo sensóriomotor, conforme o tipo de enxerto usado. Estes achados podem orientar a reabilitação de indivíduos submetidos à cirurgia de reconstrução do LCA.

DEsCRITORES: Eletromiografia; Estudo de caso; Ligamento cruzado anterior; Propriocepção

ABSTRACT: The aim of this case study was to describe electromyographic (EMG) activity of the knee stabilizing muscles in subjects having received different autografts in anterior cruciate ligament (ACL) surgical reconstruction: two subjects with anterior bone-tendon-bone graft, two with hamstring graft and two controls. Normalised EMG signal was collected during maximum voluntary isometric contraction (MVIC) of each muscle (vastus medialis obliques, vastus lateralis, semitendinosus, biceps femoris and gastrocnemius) and then under six perturbation stimuli at one-leg stance: on the ground, inclined and round board, balance board, anterior-posterior (AP) and medium-lateral (ML) rollerboard. When comparing only extensor muscles between cases, a higher percent of MVIC was found in vastus medialis obliques and vastus lateralis in the anterior graft group (during one-leg stance, inclined board, and AP roller-board), whereas in hamstring graft cases this occurred on the round and balance boards. The analysis of flexor muscles showed greater activity on balance board and $M L$ roller-board in subjects with hamstring graft. The amount of muscle activation thus may vary according to the stimulus. It may be said that muscles are most activated in subjects with anterior graft. These findings may guide rehabilitation of patients submitted to ACL reconstruction.

KEY wORDS: Anterior cruciate ligament; Case studies; Electromyography; Proprioception 


\section{INTRODUÇÃO}

O ligamento cruzado anterior (LCA) é o principal responsável pela estabilidade ântero-posterior do joelho, junto com o ligamento cruzado posterior; $50 \%$ das lesões na articulação do joelho recaem sobre o LCA ${ }^{1,2}$. As lesões ocorrem principalmente em atividades esportivas, por submeterem o ligamento a grandes tensões ${ }^{3}$.

As lesões no LCA são reparadas por cirurgia em que se procede a um enxerto para formar um neo-ligamento. A literatura aponta divergências na escolha do enxerto ideal ${ }^{3-5}$. Entre as possibilidades de enxertos autólogos, duas técnicas são mais usadas: terço médio do tendão patelar e tendão do músculo semitendinoso associado ou não ao tendão do músculo grácil ${ }^{6}$. Em uma meta-análise ficou demonstrado que as duas técnicas não apresentaram diferença estatisticamente significante quanto à estabilidade, porém sua escolha deve ser individualizada ${ }^{7}$. Tem havido aumento do uso dos tendões flexores ${ }^{8}$; um estudo nacional precursor nesse campo foi desenvolvido por Gomes e Marczyk em $1981^{9}$. A técnica de uso dos tendões flexores somente conquistou reconhecimento após o desenvolvimento de melhores placas para fixação do enxerto ${ }^{10}$.

A fisioterapia após a reconstrução cirúrgica do LCA tem objetivo inicial de controle do processo infamatório e alívio da dor, além do ganho do arco de movimento. Na fase final da fisioterapia dá-se ênfase ao recrutamento muscular, além do estímulo sensóriomotor $^{11,12}$. É importante lembrar que, mesmo ao final do tratamento, permanece com certa freqüência um deficit proprioceptivo $^{13}$. Esse deficit é devido ao LCA não apresentar apenas função biomecânica, mas também uma função sensorial proveniente dos mecanorreceptores aí presentes ${ }^{14}$.

Os mecanorreceptores do LCA têm o papel de proteção; sua excitação pode iniciar um arco reflexo que leva à contração muscular. Dessa forma a instabilidade após uma lesão do LCA não é causada somente pela estabilidade mecânica perdida: há também instabilidade funcional, devido ao menor número de mecanorreceptores ${ }^{14,15}$.

Alguns estudos já demonstraram o comportamento dos estabilizadores do joelho em indivíduos com lesão do LCA durante estímulos sensório-motores em cadeia cinética fechada ${ }^{16,17}$; contudo, a influência do tipo de enxerto usado na reconstrução quando se aplicam os estímulos sensório-motores ainda é motivo de discussão. O objetivo deste estudo foi descrever o comportamento dos músculos estabilizadores do joelho em indivíduos sem lesão e indivíduos que usaram diferentes enxertos para reconstrução do LCA enquanto realizavam exercícios de estímulo sensório-motor.

\section{METODOLOGIA}

Este é um estudo de casos, que foi previamente aprovado pelo Comitê de Ética em Pesquisa da Universidade Estadual de Londrina (CEP 038/04); todos os participantes assinaram o termo de consentimento livre e esclarecido.

\section{Participantes}

Foram selecionados seis participantes do sexo masculino (quatro com reconstrução do LCA e dois sem lesão) que realizavam atividade física moderada entre duas e três vezes por semana e apresentavam tempo de lesão até o teste entre 12 e 18 meses. Os casos que haviam se submetido a cirurgia para reconstrução do LCA tinham os seguintes critérios de inclusão: cirurgia realizada há pelo menos 12 meses, tempo de reabilitação entre 6 e 8 meses, ter realizado treinamento com estímulos sensório-motores durante o período de tratamento e recuperado amplitude de movimento normal (flexão e extensão do joelho). Os indivíduos seriam excluídos se apresentassem dor no joelho ao movimento ou repouso, derrame articular ou edema.

Nesses casos com reconstrução do LCA, dois participantes haviam recebido na cirurgia enxerto posterior com tendões quádruplos de semitendinoso e grácil (idades 24 e 33 anos; massa, $82,8 \mathrm{~kg}$ e 94,8 kg; estatura, 1,82 m e $1,84 \mathrm{~m})$; e em fora feito enxerto anterior com o terço central do tendão patelar (idades 20 e 30 anos; massa, $72 \mathrm{~kg}$ e 94,3 kg; estatura, 1,67 m e $1,67 \mathrm{~m})$. Os outros dois participantes foram indivíduos sem lesão (idades 27 e 35 anos; massa, 81,5 e 77,4 kg; estatura, 1,86 m e 1,87 m). Esses participantes tinham características morfológicas parecidas com os que haviam sido operados, porém sem história de lesão no joelho ou em membro inferior.

\section{Instrumentação}

Para caracterizar as condições dos participantes quanto ao desempenho físico foi usado o Single Leg Hop Test18 (SLH-Test) e, para avaliar a funcionalidade subjetiva do joelho, o questionário de Lysholm ${ }^{19}$. No SLH-Test, o indivíduo é solicitado a fazer, com cada membro inferior, três tentativas de um único salto à frente em apoio unipodal, calculando-se a razão entre as distâncias saltadas com cada perna. Essa caracterização do desempenho físico foi realizada apenas nos indivíduos que haviam se submetido à reconstrução do LCA, já que indivíduos da amostra controle não apresentariam alteração nesses testes.

Para obtenção dos sinais elétricos foi utilizado um eletromiógrafo de superfície com 16 canais (EMG System do Brasil) composto por um conversor analógico-digital de 12 bits, freqüência de corte com banda de 20 a $450 \mathrm{~Hz}$, ganho de amplificação total 2000x, sendo 20x no eletrodo ativo e 100x no segundo estágio de amplificação. Cada canal é acoplado a dois eletrodos ativos e um de referência. Os eletrodos foram conectados a um pré-amplificador de alta impedância (1.0 x 1012 Ohm), pré-amplificação de 20x, com razão de rejeição do modo comum de $120 \mathrm{~dB}$. Os sinais foram coletados com freqüência de amostragem de 2.000 amostras por segundo por canal, e analisados em um programa computacional de aquisição de dados (AqDados $\left.{ }^{\circledR} 5.0\right)$. 


\section{Procedimentos}

Eletrodos de superfície circulares $(\mathrm{Ag} / \mathrm{AgCl})$, com $10 \mathrm{~mm}$ de diâmetro (Meditrace ${ }^{\circledR}$ ), foram colocados em pares (distância entre eletrodos de 2 $\mathrm{cm})$ no membro inferior sobre os músculos vasto medial oblíquo (VMO), vasto lateral $(\mathrm{VL})$, semitendinoso $(\mathrm{ST})$, bíceps femoral (BF) e gastrocnêmio porção média (GAS), segundo as recomendações do Seniam (Surface EMG for a non-invasive assessment of muscles ${ }^{20}$. Realizou-se tricotomia e a limpeza do local com algodão e álcool $70 \%$ antes da colocação dos eletrodos. A impedância intereletrodos foi menor que $10 \mathrm{k} \Omega$. O eletrodo de referência foi colocado no punho contra-lateral. Foram captados os sinais elétricos do membro inferior que havia sido operado ou no membro inferior dominante, nos casos controle. Todos os indivíduos eram destros ou receberam cirurgia no membro inferior dominante.

Coletaram-se em seguida os sinais elétricos durante contrações isométricas voluntárias máximas (CIVM). Para a coleta da CIVM dos músculos extensores do joelho (vasto medial oblíquo e vasto lateral) foi utilizada uma mesa extensora com o paciente sentado, quadril a $60^{\circ}$ de flexão e o joelho em flexão de $30^{\circ}$. Para os músculos flexores (semitendinoso e bíceps femoral) o paciente se posicionava de pé em uma mesa flexora com as mãos apoiadas e anteparo na região anterior da coxa; nessa postura uma resistência no sentido súpero-inferior era aplicada sobre o terço distal posterior da perna com $90^{\circ}$ de flexão de joelho. Para o gastrocnêmio, a CIVM foi realizada com o paciente em pé sob uma barra transversal fixa; o voluntário fazia força, com os joelhos em extensão, no sentido de elevar a barra com os ombros.

Após a coleta das CIVM, foram captados os sinais elétricos durante os exercícios de estímulos sensóriomotores. A ordem dos estímulos foi aleatória, evitando fadiga no último aparelho. Foi permitido aos participantes realizar um leve treinamento nos exercícios. Após isso, houve um minu- to de captação do sinal pelo eletromiógrafo. Foram descartados os dez primeiros e os dez últimos segundos. Foram feitos seis exercícios: 1 - apoio unipodal em solo (superfície plana); 2 - apoio unipodal sobre uma prancha inclinada (base retangular de madeira com 100 $\mathrm{cm}$ de comprimento por $50 \mathrm{~cm}$ de largura, duas estruturas semi-ovais com $50 \mathrm{~cm}$ de comprimento, $3 \mathrm{~cm}$ de largura e $6 \mathrm{~cm}$ de raio posicionadas na base em cada extremidade do equipamento); 3 - apoio unipodal sobre uma prancha redonda (base de madeira, com $50 \mathrm{~cm}$ de diâmetro e uma semiesfera fixada na face inferior com 13 $\mathrm{cm}$ de diâmetro e 6,5 cm de raio); 4 apoio unipodal sobre um balancinho (estrutura de alumínio, com base de sustentação de $54 \mathrm{~cm}$ de comprimento por $42 \mathrm{~cm}$ de largura e, nas extremidades, quatro barras com $40 \mathrm{~cm}$ de altura ligadas à base sustentação; quatro correntes com $32 \mathrm{~cm}$ de comprimento, fixadas nessas barras, sustentam uma base suspensa de apoio para o pé - 10 $\mathrm{cm}$ de altura, $31 \mathrm{~cm}$ de comprimento e $15 \mathrm{~cm}$ de largura); 5 e 6 - apoio unipodal sobre um rollerboard (base quadrada de madeira com $42 \mathrm{~cm}$ de lado, altura de $10 \mathrm{~cm}$ e quatro rodas fixadas nos cantos inferiores); esse equipamento foi utilizado no deslocamento ântero-posterior e médiolateral (Figura 1). Em cada um desses estímulos o indivíduo não usou apoio para as mãos ou para o membro inferior contralateral. O joelho do membro inferior de apoio foi posicionado a $30^{\circ}$ de flexão para evitar a trava articular de extensão e o individuo era estimulado verbalmente a manter essa posição (em isometria), bem como seu centro de gravidade, numa posição o mais estável possível. Para a manutenção dos $30^{\circ}$ de flexão do joelho, um dos avaliadores mantinha um goniômetro próximo à articulação testada, dando ao sujeito feedback sobre seu posicionamento.

\section{Análise do sinal}

Os sinais eletromiográficos foram retificados e filtrados pelo programa AcqKnowledge 3.7.1 (Biopac System
Inc.). O método usado para a análise quantitativa da amplitude do potencial elétrico foi o da raiz quadrada da média (RMS - root mean square), expresso em microvolts $(\mu \mathrm{V})$. O valor da CIVM foi usado para normalizar os sinais obtidos durante os estímulos sensório-motores.

\section{Análise dos dados}

As variáveis funcionais são apresentadas de forma descritiva. As variáveis eletromiográficas (\% CIVM) são apresentadas com média e desvio padrão, em forma de tabela e não foram comparadas entre si por se tratar de um relato de casos.

\section{RESULTADOS}

O primeiro participante com reconstrução do enxerto posterior apresentou resultado do SLH-Test de 93\% no membro operado em relação ao membro contralateral e 89 pontos na escala de Lysholm. O segundo participante com enxerto posterior obteve $99 \%$ no SLH-Test e 95 pontos na escala de Lysholm.

O primeiro participante com reconstrução usando enxerto patelar apresentou $90 \%$ em relação ao membro contralateral no SLH-Test e 85 pontos na escala de Lysholm. Já o segundo participante com esse enxerto obteve $103 \%$ em relação ao membro contralateral no do SLH-Test de e 76 pontos no Lysholm. Os resultados das respostas de cada músculo aos diferentes estímulos são apresentados nas Tabelas 1 a 5 .

A maior atividade elétrica do músculo VMO foi apresentada nos exercícios de solo, prancha inclinada e rollerboard AP nos casos de reconstrução do LCA com enxerto patelar. Nesse músculo, os casos controle apresentaram menor atividade em todos os estímulos, conforme Tabela 1. No músculo VL a maior atividade elétrica é encontrada no solo, prancha inclinada, balancinho e rollerboard AP, nos casos de reconstrução do LCA com enxerto patelar, conforme Tabela 2 . 


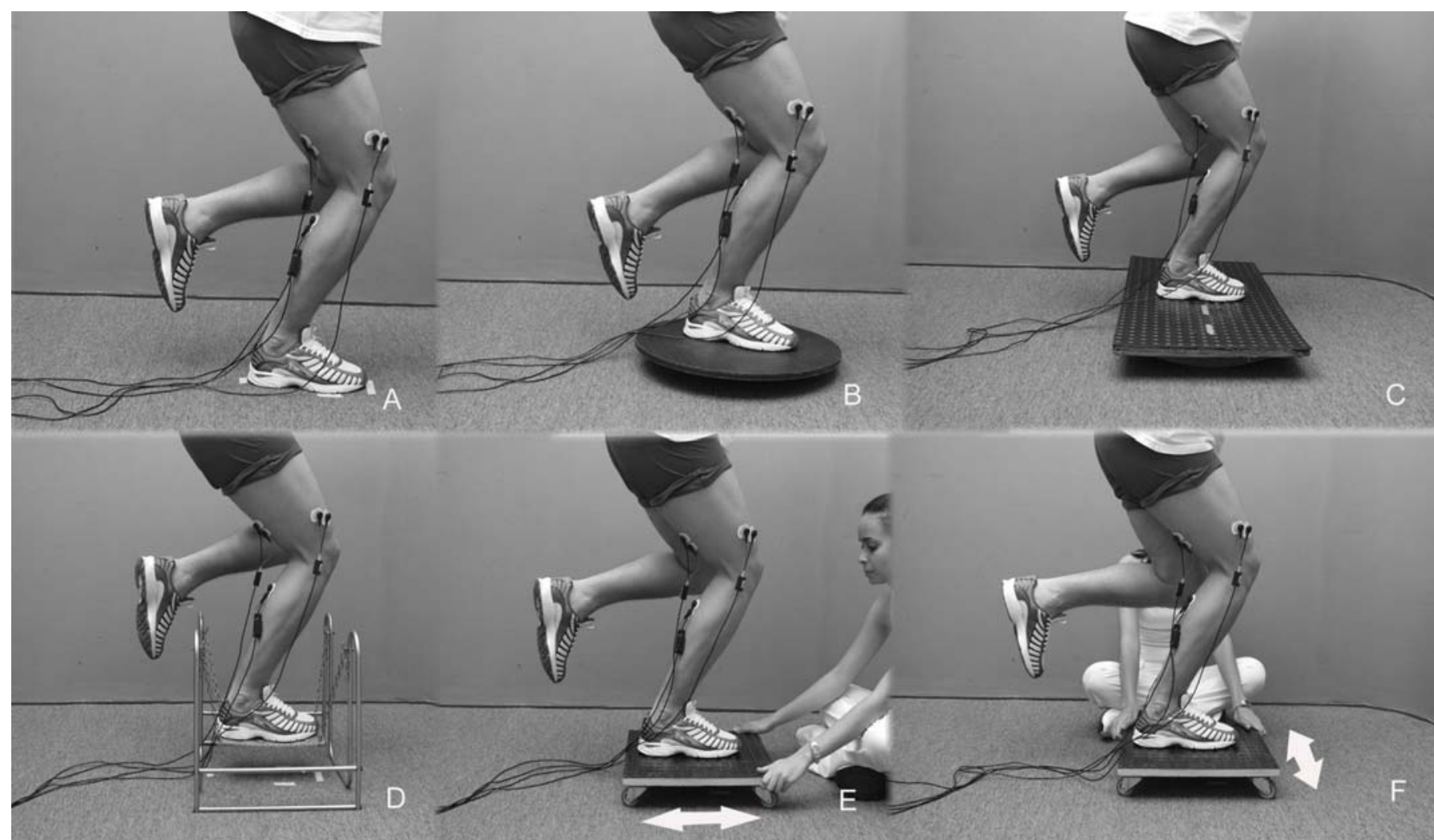

Figura 1 Ilustração dos exercícios de estímulos sensório-motores em apoio unipodal. A: no solo; B: sobre prancha redonda; C: sobre prancha inclinada; D: em balancinho; E: em rollerboard, com deslocamento ântero-posterior; F: em rollerboard, com deslocamento médio-lateral

Quanto ao músculo semitendinoso, a maior ativação foi encontrada na prancha redonda, balancinho e rollerboard ML em um dos casos de reconstrução do LCA com enxerto posterior. Nesse músculo, os casos controle apresentaram a menor atividade elétrica em todos os estímulos, com exceção do rollerboard AP (Tabela 3).

Para o músculo bíceps femoral, em todos os estímulos, a maior atividade elétrica foi apresentada pelos casos de reconstrução do LCA usando enxerto anterior (Tabela 4). No músculo gastrocnêmio, a maior atividade elétrica ocorreu nos casos de reconstrução do LCA com enxerto patelar. Houve discreta diferença no estímulo do balancinho entre casos com enxerto patelar e posterior (Tabela 5).

\section{DISCUSSÃO}

Os casos de reconstrução do LCA com enxerto posterior apresentaram um índice excelente no Single Leg
Hop Test (maior que $85 \%)^{18}$ e na escala de Lysholm. Os casos com enxerto patelar também apresentaram um índice excelente no SLH-Test, porém na escala de Lysholm apresentaram menor pontuação do que os casos com enxerto posterior, talvez devido à morbidade causada pela agressão ao mecanismo extensor.

Optou-se por realizar o trabalho em cadeia cinética fechada, pois oferece grande estímulo sensório-motor e estabilização dinâmica por meio da cocontração muscular ${ }^{16}$. Observa-se de um modo geral que a musculatura extensora do joelho apresentou atividade elétrica superior à musculatura flexora. Talvez esses valores tenham sido encontrados neste estudo devido à permanência em $30^{\circ}$ de flexão do joelho em apoio unipodal durante o teste, pois o joelho sai da posição de trava articular quando está totalmente estendido e a musculatura extensora entra em melhor posição para contração. Resultados semelhantes, relatados por Heller et al. ${ }^{17}$, apontam uma maior ativação elétrica dos músculos extensores em relação aos flexores durante estímulo sensório-motor (deslocamento lateral) em cadeia cinética fechada (com variação na angulação do joelho).

Quando se observa apenas a musculatura extensora do joelho, o músculo VMO apresenta maior ativação nos indivíduos com reconstrução, independente do enxerto usado, quando comparado aos indivíduos sem lesão. Porém o VL da amostra com enxerto posterior tem menor atividade comparado ao dos indivíduos sem lesão nos exercícios de solo, prancha inclinada, prancha redonda, rollerboard AP e rollerboard ML.

No estudo de Ciccotti et al. ${ }^{16}$ os sujeitos com lesão do LCA que não fizeram reconstrução apresentaram maior atividade elétrica do VL quando comparados a sujeitos controle e a sujeitos com reconstrução do ligamento, quando esses sujeitos realizavam atividades funcionais. Isso indica a variabilidade de ativação do mecanis- 
Tabela 1 Porcentagem da CIVM do músculo vasto medial oblíquo sob diferentes estímulos sensório-motores

\begin{tabular}{lcccccc}
\hline Sujeito & Solo & Pr. inclinada & Pr. redonda & Balancinho & Rollerboard AP & Rollerboard ML \\
\hline Controle 1 & 42,6 & 53,6 & 73,2 & 59,3 & 36,7 & 54,0 \\
Controle 2 & 47,2 & 61,4 & 61,6 & 70,5 & 46,1 & 68,2 \\
Enx Post 1 & 70,2 & 73,0 & 92,2 & 90,2 & 72,2 & 78,6 \\
Enx Post 2 & 72,0 & 82,0 & 99,4 & 90,4 & 80,4 & 87,2 \\
Enx Ant 1 & 100,7 & 92,2 & 78,0 & 77,0 & 90,2 & 63,2 \\
Enx Ant 2 & 97,1 & 103,0 & 94,0 & 97,0 & 103,2 & 73,4
\end{tabular}

Pr. = prancha; $\mathrm{AP}=$ ântero-posterior; $\mathrm{ML}=$ médio-lateral, Enx = Enxerto, Ant = Anterior, Post = Posterior

Tabela 2 Porcentagem da CIVM do músculo vasto lateral sob diferentes estímulos sensório-motores

\begin{tabular}{lcccccc}
\hline Sujeito & Solo & Pr. inclinada & Pr. redonda & Balancinho & Rollerboard AP & Rollerboard ML \\
\hline Controle 1 & 48,0 & 64,0 & 79,4 & 87,2 & 57,0 & 67,3 \\
Controle 2 & 53,0 & 79,0 & 86,0 & 75,4 & 50,2 & 74,1 \\
Enx Post 1 & 43,7 & 43,0 & 62,4 & 81,2 & 45,2 & 36,5 \\
Enx Post 2 & 46,1 & 48,2 & 82,0 & 88,0 & 48,4 & 46,3 \\
Enx Ant 1 & 92,3 & 94,1 & 72,2 & 87,4 & 92,0 & 53,0 \\
Enx Ant 2 & 100,1 & 105,3 & 88,2 & 97,2 & 101,2 & 64,0
\end{tabular}

Pr. = prancha; $\mathrm{AP}=$ ântero-posterior; $\mathrm{ML}=$ médio-lateral, Enx = Enxerto, Ant = Anterior, Post = Posterior

Tabela 3 Porcentagem da CIVM do músculo semitendinoso sob diferentes estímulos sensório-motores

\begin{tabular}{lcccccc}
\hline Sujeito & Solo & Pr. inclinada & Pr. redonda & Balancinho & Rollerboard AP & Rollerboard ML \\
\hline Controle 1 & 7,2 & 9,7 & 21,2 & 15,0 & 20,0 & 8,5 \\
Controle 2 & 11,2 & 12,1 & 25,6 & 19,2 & 18,4 & 15,3 \\
Enx Post 1 & 10,0 & 14,5 & 39,5 & 37,6 & 12,1 & 41,2 \\
Enx Post 2 & 19,0 & 17,3 & 54,1 & 50,2 & 21,3 & 51,4 \\
Enx Ant 1 & 41,3 & 26,0 & 35,6 & 40,5 & 33,4 & 33,1 \\
Enx Ant 2 & 42,3 & 34,0 & 46,2 & 42,3 & 42,2 & 44,1
\end{tabular}

Pr. = prancha; $\mathrm{AP}=$ ântero-posterior; $\mathrm{ML}=$ médio-lateral, Enx $=$ Enxerto, Ant $=$ Anterior, Post $=$ Posterior

Tabela 4 Porcentagem da CIVM do músculo bíceps femoral sob diferentes estímulos sensório-motores

\begin{tabular}{lcccccc}
\hline Sujeito & Solo & Pr. inclinada & Pr. redonda & Balancinho & Rollerboard AP & Rollerboard ML \\
\hline Controle 1 & 9,2 & 12,1 & 32 & 16,2 & 31,1 & 13,0 \\
Controle 2 & 15,6 & 20,3 & 51 & 30,4 & 34,7 & 15,0 \\
Enx Post 1 & 6,4 & 4,7 & 10,1 & 42,2 & 5,7 & 40,0 \\
Enx Post 2 & 8,2 & 5,3 & 6,1 & 50,6 & 5,1 & 54,4 \\
Enx Ant 1 & 51,3 & 50,0 & 92,2 & 53,7 & 65,5 & 61,3 \\
Enx Ant 2 & 60,5 & 66,0 & 96,6 & 56,1 & 72,1 & 67,3
\end{tabular}

Pr. = prancha; $\mathrm{AP}=$ ântero-posterior; $\mathrm{ML}=$ médio-lateral, Enx $=$ Enxerto, Ant $=$ Anterior, Post $=$ Posterior

Tabela 5 Porcentagem da CIVM do músculo gastrocnêmio sob diferentes estímulos sensório-motores

\begin{tabular}{lcccccc}
\hline Sujeito & Solo & Pr. inclinada & Pr. redonda & Balancinho & Rollerboard AP & Rollerboard ML \\
\hline Controle 1 & 19,8 & 19,9 & 38,1 & 32,9 & 28 & 14,4 \\
Controle 2 & 11,4 & 33,9 & 40,3 & 37,1 & 39 & 23,2 \\
Enx Post 1 & 13,4 & 13,4 & 23,5 & 86,1 & 14,3 & 62,0 \\
Enx Post 2 & 14,2 & 15,8 & 41,3 & 109,9 & 17,3 & 73,4 \\
Enx Ant 1 & 77,1 & 60,2 & 77,5 & 115,1 & 68,4 & 92,0 \\
Enx Ant 2 & 112,1 & 46,4 & 70,3 & 84,7 & 73,4 & 74,2
\end{tabular}

Pr. = prancha; $\mathrm{AP}=$ ântero-posterior; $\mathrm{ML}=$ médio-lateral, $\mathrm{Enx}=$ Enxerto, Ant $=$ Anterior, Post $=$ Posterior 
mo extensor conforme o tipo de enxerto usado e reconstrução do ligamento.

A amostra com enxerto patelar obteve, em geral, alta porcentagem da CIVM dos músculos VMO e VL; contudo, no exercício de rollerboard ML isso não foi tão evidente, talvez pelo fato de esse estímulo não exigir tanto deslocamento ântero-posterior. Essa grande ativação do mecanismo extensor apresentado pelos casos com enxerto patelar, quando comparados aos outros casos, não se repetiu na amostra com enxerto posterior, talvez pela morbidade menor causada ao mecanismo extensor quando a cirurgia é realizada com os tendões flexores.

Quando analisados apenas os músculos flexores em todos os casos, os participantes com enxerto patelar tiveram maior atividade elétrica do músculo bíceps femoral em todos os estímulos. Nos casos com enxerto dos tendões semitendinoso e grácil, essa mesma relação não foi encontrada, provavelmente devido ao uso dos tendões desses músculos como enxerto.

Quando se observa o músculo semitendinoso, a atividade elétrica das amostras com reconstrução (independente do tipo de enxerto) é sempre maior quando comparada à da amostra controle, com exceção dos pacientes com reconstrução usando enxerto posterior no estímulo do rollerboard AP.

Esperava-se que o músculo gastrocnêmio tivesse sido altamente recrutado na amostra com enxerto posterior, com a função de auxiliar o controle da flexão do joelho, devido à falta dos tendões do grácil e semitendinoso. Entretanto, sua maior ativação foi nos casos de enxerto patelar. Talvez isso não tenha ocorrido devido à flexão de $30^{\circ}$ do joelho durante os estímulos usados, o que pode ter colocado o gastrocnêmio em posição desfavorável para seu desempenho.

Parisaux et al. ${ }^{21}$ relataram, por meio da avaliação isocinética, o deficit de força apresentado pela musculatura flexora do joelho em indivíduos em que foi utilizado o tendão do músculo semitendinoso associado ao tendão do músculo grácil para reconstrução do LCA. Alguns autores, porém, contradizem esse deficit de força após seis meses da reconstrução e ainda relatam um progressivo aumento da força de flexão após seis meses de reabilitação ${ }^{22-24}$. Quando se estudam as atividades funcionais pela eletromiografia de superfície, poucos estudos levam em consideração as alterações anatômicas decorrentes de cada tipo de reconstrução do LCA. Talvez esse aumento progressivo de força de flexão do joelho possa alterar as estratégias de ativação muscular e conseqüentemente mudar os mecanismos de co-contração no membro inferior.

Quanto ao controle motor após a lesão do LCA, Chmielewski et al. ${ }^{25}$ consideram o estímulo sensório-motor essencial para a normalização da cocontração muscular e sinergia entre extensores e flexores. Portanto, talvez essa diferença na atividade elétrica entre extensores e flexores encontrada neste estudo possa representar algum deficit funcional de co-contração exacerbado durante os estímulos sensóriomotores, sem um treinamento prévio.

Talvez a atividade elétrica reduzida do bíceps femoral encontrada neste estudo se deva ao tecido cicatricial formado no local, além do reposicionamento do ventre muscular após a retirada de seus tendões para uso como enxerto. Os sujeitos que foram submetidos à cirurgia usando enxerto patelar parecem apresentar maior ativação elétrica nos estímulos do solo, prancha inclinada e rollerboard AP. Enquanto, nos sujeitos submetidos à cirurgia que recorreu aos tendões flexores, os estímulos de balancinho, prancha redonda e rollerboard ML parecem provocar maior ativação elétrica. O músculo gastrocnêmio apresenta grande ativação durante o estímulo do balancinho, independente do tipo de enxerto usado.

A maior atividade elétrica pode sugerir que o estímulo é mais intenso ou talvez mais difícil para o paciente e, dessa forma, o fisioterapeuta pode escolher primeiro os estímulos de menor atividade elétrica em uma fase inicial de pós-operatório e, depois, evoluir para os estímulos que deman- dam maior atividade elétrica. Isso talvez permita graduar o tipo de estímulo, dependendo do tipo de cirurgia a que o paciente foi submetido.

A principal limitação deste relato descritivo de casos é a amostra limitada, que não permite testar uma hipótese. Acredita-se que este estudo tenha implicações para a prática clínica, pois os fisioterapeutas devem estar atentos quanto à importância dos exercícios sensório-motores individualizados para cada tipo de enxerto usado na cirurgia para reconstrução do LCA, devido ao possível deficit de recrutamento dos músculos estabilizadores (alteração na co-contração) característico de cada procedimento cirúrgico. Espera-se que estudos futuros possam ser feitos com uma amostra adequada e com poder para comparar resultados entre os diferentes tipos de cirurgia.

\section{CONCLUSÃO}

Os casos submetidos às cirurgias de reconstrução apresentam maior atividade elétrica do que os casos controle, independente do estímulo sensóriomotor. E, durante os exercícios de estímulos, a ativação muscular parece variar conforme o tipo de enxerto usado. Nos casos com enxerto patelar, os estímulos de solo, prancha inclinada e rollerboard AP provocaram maior atividade elétrica nos músculos VL e VMO. Nos casos com enxerto posterior, os estímulos da prancha redonda e balancinho resultaram em maior ativação do VMO e VL. Os casos com enxerto patelar apresentaram maior atividade muscular que os casos com enxerto posterior ou controle, sob todos os estímulos. Dentre os estímulos, o balancinho provocou a menor variação de ativação muscular nos sujeitos com reconstrução de LCA mas, ainda assim, essa ativação ainda foi maior do que nos casos controle.

Os fisioterapeutas devem considerar esses dados ao proceder à reabilitação de pacientes submetidos à cirurgia de reconstrução do LCA - considerando no entanto sua limitação, devido ao pequeno número de casos analisados. 


\section{REFERENNCIAS}

1 Duthon VB, Barea C, Abrassart S, Fasel JH, Fritschy D, Menetrey J. Anatomy of the anterior cruciate ligament. Knee Surg Sports Traumatol Arthrosc. 2006;14:204-13.

2 Bollen S. Ligament injuries of knees: limping forward? Br J Sports Med. 1998;2:82-4.

3 Forssblad M. ACL reconstruction: patellar tendon versus hamstring grafts: economical aspects. Knee Surg Sports Traumatol Arthrosc. 2006;14:539-41.

4 Gobbi A, Francisco R. Factors affecting return to sports after anterior cruciate ligament reconstruction with patellar tendon and hamstring graft: a prospective clinical investigation. Knee Surg Sports Traumatol Arthrosc. 2006;14:1021-8.

5 Ibrahim SA, AI-Kussary IM, AI-Misfer AR. Clinical evaluation of arthroscopically assisted anterior cruciate ligament reconstruction: patellar tendon versus gracilis and semitendinosus autograft. Arthroscopy. 2005;21:412-7.

6 Gali JC, Adad MAH, Mod MSB. Reconstrução do ligamento cruzado anterior com tendões flexores quádruplos e parafusos de interferência metálicos. Rev Bras Ortop. 2002;37:240-6.

7 Goldblatt JP, Fitzsimmons SE, Balk E, Richmond JC. Reconstruction of the anterior cruciate ligament: meta-analysis of patellar tendon versus hamstring tendon autograft. Arthroscopy. 2005;21:791-803.

8 Bilko T, Paulos L, Feagin JJ, Lambert K, Cunninghan $\mathrm{H}$. Current trends in repair and rehabilitation of complete (acute) anterior cruciate ligament injuries: analysis of 1984 questionnaires completed by ACL study group. Am J Sports Med. 1986;14:143-7.

9 Gomes JLE, Marczyk LRS. Reconstrução dos ligamentos cruzados do joelho com o tendão duplo do semitendinoso. Rev Bras Ortop. 2004;39:137-46.

10 Camanho GL, Andrade MH. Estudo comparativo da reabilitação dos pacientes submetidos à reconstrução do ligamento cruzado anterior com enxertos do terço médio do tendão patelar e dos tendões dos músculos flexores mediais do joelho. Rev Bras Ortop. 1999;34:513-8.
11 Thomson LC, Handoll HHG, Cunningham A, Shaw PC. Physiotherapist-led programmes and interventions for rehabilitation of anterior cruciate ligament, medial collateral ligament and meniscal injuries of the knee in adults. Cochrane Database of Systematic Reviews 2002; Issue 2. Art. No. CD001354.

12 Sampaio TCFVS, Souza JMGS. Reeducação proprioceptiva nas lesões do ligamento cruzado anterior do joelho. Rev Bras Ortop. 1994;29:303-8.

13 Bonfim RT, Paccola CAJ. Propriocepção após reconstrução do ligamento cruzado anterior usando ligamento patelar homólogo e autólogo. Rev Bras Ortop. 2000;35:194-201.

14 Barrett DS. Proprioception and function after anterior cruciate reconstruction. J Bone Joint Surg Br. 1991;73:833-7.

15 Reider B, Arcand MA, Diehl LH, Mroczek K, Abulencia A, Stroud C. Proprioception of the knee before and after anterior cruciate ligament reconstruction. Arthroscopy. 2003;19:2-12.

16 Ciccotti MG, Kerlan RK, Perry J, Pink M. An electromyographic analysis of the knee during functional activities, II: the anterior cruciate ligament-deficient and -reconstructed profiles. Am J Sports Med. 1994;22:651-8.

17 Heller BM, Pincivero DM. The effect of ACL injury on lower extremity activation during closed kinetic chain exercise. J Sports Med Phys Fitness. 2003;43:180-8.

18 Noyes FR, Barber SD, Mangine RE. Abnormal lower limb symmetry determined by function hop tests after anterior cruciate ligament rupture. Am J Sports Med. 1991;19:513-8.

19 Peccin MS. Questionário específico para os sintomas do joelho "Lysholm Knee Scoring Scale" - tradução e validação para a língua portuguesa. Acta Ortop Bras. 2006;14:268-72.

20 Hermens HJ, Freriks B, Disselhorst-Klug C, Rau G. Development of recommendations for SEMG sensors and sensor placement procedures. J Electromyogr Kinesiol. 2000;10:361-74. 


\section{Referências (cont.)}

21 Parisaux JM, Boileau P, Desnuelle C. Isokinetic evaluation of knee flexor muscle after anterior cruciate ligament reconstruction using gracilis and semitendinosus tendon graft. Rev Chir Orthop. 2004;90:33-9.

22 Anderson JL, Lamb SE, Barker KL, Davies S, Dodd CA, Beard DJ. Changes in muscle torque following anterior cruciate ligament reconstruction: a comparison between hamstrings and patella tendon graft procedures on 45 patients. Acta Orthop Scand.

2002;73:546-52.
23 Marcacci M, Zaffagnini S, lacono F, Vascellari A, Loreti I, Kon E, et al. Intra- and extra-articular anterior cruciate ligament reconstruction utilizing autogeneous semitendinosus and gracilis tendons: 5-year clinical results. Knee Surg Sports Traumatol Arthrosc. 2003;11:2-8.

24 Kobayashi A, Higuchi H, Terauchi M, Kobayashi F, Kimura $\mathrm{M}$, Takagishi K. Muscle performance after anterior cruciate ligament reconstruction. Int Orthop. 2004;28:48-51.

25 Chmielewski TL, Hurd W], Rudolph KS, Axe MJ, SnyderMackler L. Perturbation training improves knee kinematics and reduces muscle co-contraction after complete unilateral anterior cruciate ligament rupture. Phys Ther. 2005;8:740-52. 